\section{Effort Dyspnea, if it is not Asthma? An Omega Epiglottis Case Report}

Mustafa Ilker Inan ${ }^{1 *}$, Ferhat Cuce ${ }^{2}$ and Hakan Genc ${ }^{3}$

${ }^{1}$ Girne Military Hospital, Department of Pulmonary Medicine, Girne, Turkish Republic of North Cyprus, Turkey

${ }^{2}$ Ankara Mevki Military Hospital, Department of Radiology, Ankara, Turkey

3/sparta Military Hospital, Department of Ear, Nose and Throat, Isparta, Turkey

*Corresponding author: Mustafa Ilker Inan, Girne Military Hospital, Department of Pulmonary Medicine, Girne, Turkish Republic of North Cyprus, Turkey, Tel: +905488805054, +905052795054; E-mail: drilkerinan@yahoo.com

Rec date: Apr 25, 2016; Acc date: June 14, 2016; Pub date: June 20, 2016

Copyright: (c) 2016 Inan MI, et al. This is an open-access article distributed under the terms of the Creative Commons Attribution License, which permits unrestricted use, distribution, and reproduction in any medium, provided the original author and source are credited.

\section{Abstract}

Asthma is a chronic inflammatory disorder of the airways which is characterized by recurrent episodes of airway obstruction, associated with airway hyper responsiveness that leads to symptoms of dyspnea, wheezing, chest tightness, and/or coughing. Omega shaped epiglottis is a type of laryngomalacia which is a result of curling on itself of the epiglottis because of shortened aryepiglottic folds.

Keywords: Asthma; Dyspnea; Omega epiglottis; Spirometry; Stridor

\section{Introduction}

Asthma is a chronic inflammatory disorder of the airways which is characterized by recurrent episodes of airway obstruction, associated with airway hyper responsiveness that leads to symptoms of dyspnea, wheezing, chest tightness, and/or coughing. These variable symptoms can be triggered by the factors like exercise, irritant or allergen exposure, whether changings or respiratory infections. Such symptoms of effort and exertional dyspnea are not uncommonly attributed to asthma without investigating other possible causes. In late adolescent patients, rare conditions of obstructive upper airway pathologies like laryngomalacia, omega epiglottis should be considered in differential diagnosis.

\section{Case Report}

A 20-year-old male patient presented to our pulmonary clinic with the symptom of dyspnea which was worsened with effort and exertion since his childhood. He stated that he had been followed with the diagnosis of asthma for 7 years. He had received different bronchodilator drug therapies that contain inhaled corticosteroids, short and long acting $\beta 2$ agonists and montelukast, but he did not get benefit and his complaints had so far continued. In his physical examination; inspiratory stridor was heard and breath sounds were normal on auscultation. Routine blood tests and chest X-ray were normal. In pulmonary function test (PFT) expiratory flow-volume curve was evaluated as normal and in inspiratory flow-volume curve a shallow flattening was detected (Figure 1). FEV1, FEV1/FVC and FVC values were normal. PFT result was concordant with variable extra thoracic airway obstruction and flexible fiber optic laryngoscopy was performed.

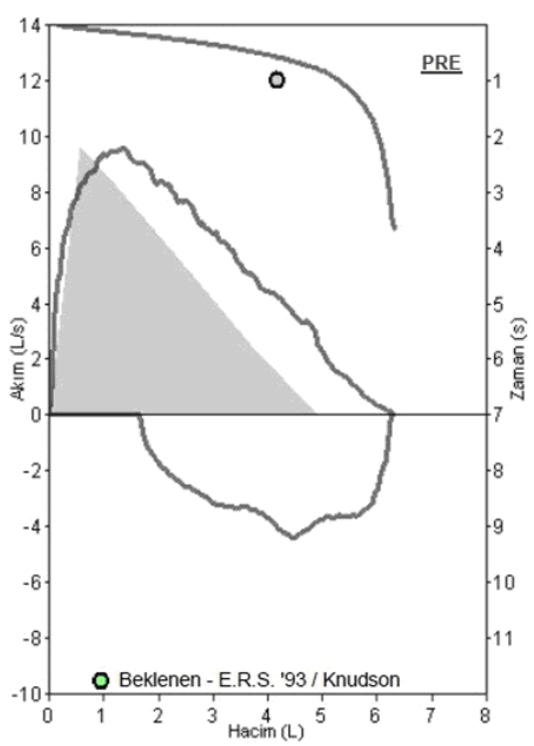

Figure 1: Spirometry: a shallow flattening in inspiratory flowvolume curve.

Laryngoscopy demonstrated an omega-shaped epiglottis, posterior prolapse of epiglottis, short aryepiglottic folds and no visualization of vocal folds. In order to evaluate other glottic and subglottic etiologies; CT virtual bronchoscopy was performed (Figures 2 and 3 ). 


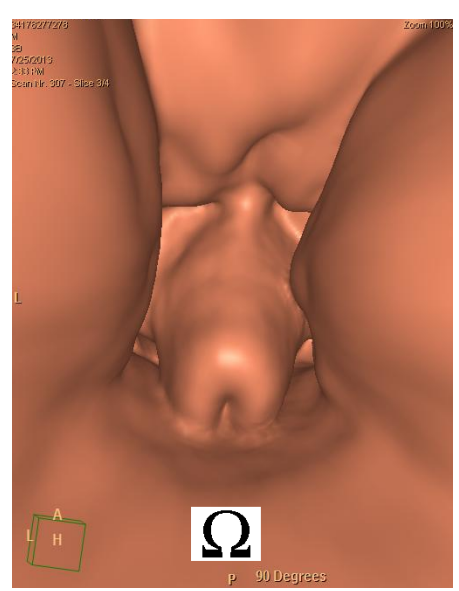

Figure 2: CT virtual bronchoscopy: the epiglottis is curled on itself and it looks like "omegashaped".

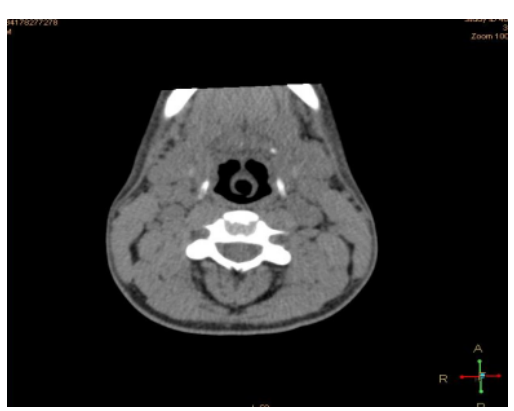

Figure 3: Axial image of epiglottis in non-contrast CT: the central part of the epiglottis is not seen because of the curl.

\section{Discussion}

Omega shaped epiglottis is a type of laryngomalacia which is a result of curling on itself of the epiglottis because of the shortened aryepiglottic folds. Laryngomalacia is the most common congenital laryngeal abnormality and is the most common cause of stridor in children, in which the soft, immature cartilage of the upper larynx (above the vocal cords) collapses inward during inhalation, causing airway obstruction and inspiratory stridor. Laryngomalacia can also occur in late years. Clinical recognition is important to avoid misdiagnosis and overtreatment as severe refractory asthma. Because adverse effects from inappropriate use of asthma medication, in particular systemic corticosteroids, are frequent and carry significant morbidity [1,2]. Exercise-induced asthma is sometimes difficult to symptomatically differentiate from exercise-related obstruction in the upper airways. The position of the symptoms and respiratory sounds in the respiratory cycle is important for differentiation of these two pathologies. Thus, airway obstruction inside the thoracic cage, as in asthma, produces principally expiratory symptoms and respiratory sounds like rhonchus or wheezing, while obstruction outside the thoracic cage produces inspiratory symptoms and stridor [2,3]. In the absence of other symptoms of asthma or lack of response to conventional asthma therapy, dyspnea on exertion most likely may result from other causes. A careful history, attention to the nature of the existing respiratory sounds, accurate spirometry assessment, flexible fiber optic laryngoscopy, and CT virtual bronchoscopy provide useful data to sort out complex cases such as the present patient, and spare patients from undergoing unnecessary and inappropriate treatment [4]. Common obstructive upper airway pathology is vocal cord dysfunction (VCD) that should be considered in differential diagnosis. VCD can also coexists with asthma and intensify present asthma symptoms. VCD occurs by involuntary paradoxical movement of the vocal folds especially by their adduction during inspiration. Laryngoscopy is the gold standart technique for definitive diagnosis of $\operatorname{VCD}[5,6]$.

CT broncopscopy, also known as virtual broncoscopy, uses low dose radiation to obtain an interior view of the larynx and trachea on CT scanning. The cross-sectional images of CT can be reformatted in multiple planes, and can even generate three-dimensional images by using virtual endoscopy software. CT virtual endoscopy has been used to evaluate pathologic processes of nasopharynx, larynx and tracheobronchial tree [7]. The benefits of this technique include noninvasive diagnostic surveillance and preoperative planning. The basic pathology in this case is an anatomical abnormality of the supraglottic area and the treatment of this disease consists of surgical procedures. The goal of the surgical procedure is to open the collapsing larynx by releasing the shortened aryepiglottic folds and removing the redundant supra-arytenoid tissue [8]. Supraglottoplasty is an effective procedure in the treatment of this disease by means of enlarging the laryngeal lumen [9]. We recommended our patient the supraglottoplasty but he did not accept this surgical treatment.

\section{References}

1. Ayari S, Aubertin G, Girschig H, Van Den Abbeele T, Mondain M (2012) Pathophysiology and diagnostic approach to laryngomalacia in infants. Eur Ann Otorhinolaryngol Head Neck Dis 129: 257-263.

2. Lim K, Li JT (2011) Exertional dyspnea and inspiratory stridor of 2 years' duration: a tale of 2 wheezes. J Allergy Clin Immunol 128: 1135-1136.

3. Røksund OD, Heimdal JH, Olofsson J, Maat RC, Halvorsen T (2014) Larynx during exercise: the unexplored bottleneck of the airways. Eur Arch Otorhinolaryngol 272: 2101-2109.

4. Weinberger M, Abu-Hasan M (2007) Pseudo - asthma: When cough, Wheezing, and Dyspnea are Not Asthma. Pediatrics 120: 855-864.

5. Pinto LH, Aun MV, Cukier-Blaj S, Stelmach R, Cukier A, et al. (2016) Vocal cord dysfunction diagnosis may be improved by a screening check list. Allergol Int. 65: 180-185.

6. Bardin PG, Low K, Ruane LE (2015) Vocal cord dysfunction: asking the right questions. Clin Exp Allergy 45: 1374-1375.

7. Luccichenti G, Cademartiri F, Fecci L, Carbognani P, Rusca M, et al. (2003) Nonneoplastic tracheal lesions: comparison between virtual CT endoscopy and fiberopticbronchoscopy. Radiol Med.106: 147-153.

8. Thompson DM (2010) Laryngomalacia: factors that influence disease severity and outcomes of management. Curr Opin Otolaryngol Head Neck Surg 18: 564-570.

9. Pinto JA, Wambier H, Mizoguchi EI, Gomes LM, Kohler R, et al. (2013)Surgical treatment of severe laryngomalacia: a retrospective study of 11 case. Braz J Otorhinolaryngol 79: 564-568. 